\title{
The Use of Exponential Sums in Step by Step Integration
}

Introduction. In the step by step numerical open integration of a system of ordinary differential equations

$$
\dot{z}_{i}=f_{i}\left(z_{1}, \cdots, z_{n}, t\right)
$$

$f_{i}\left(z_{1}, \cdots, z_{n}, t\right)$ is regarded as a function of $t, F(t)$, whose values are known at $t, t-h, \cdots, t-(n-1) h$ and for which $\int_{t}^{t+h} F(\tau) d \tau$ is desired. It is customary to assume that $F(t)$ is a polynomial of degree $n-1$ whose coefficients are determined by the given values of $F$, i.e., the values at $t, t-h, \cdots, t-(n-1) h .^{1}$ If the polynomial $F(t)$ is specified by these conditions, then the desired integral is a linear combination of the values $F(t), F(t-h), \cdots, F(t-(n-1) h)$.

GREENwOOD $^{2}$ has pointed out that the polynomial assumption for $F(t)$ may not always be the most desirable one. In particular it might be assumed that $F(t)$ is a linear sum of exponentials and still obtain that the integral is a linear combination of the values $F(t), F(t-h), \cdots, F(t-(n-1) h)$ with coefficients independent of $t$. The authors also were led to this conclusion by their own previous work. ${ }^{3}$

Sections 1-5 constitute a discussion of practical procedures and contain a description of the results obtained. Sections 6-9 contain the proofs of the results. The present discussion is for an individual step. The final total error effect on the solution and stability considerations is not given.

1. In an open integration procedure, the expression

$$
h\left(a_{0} F(t)+a_{1} F(t-h)+\cdots+a_{n-1} F(t-(n-1) h)\right)
$$

is used to approximate $\int_{t}^{t+h} F(\tau) d \tau$. The error $e(t)$ is given by

$$
\begin{aligned}
e(t)=h\left(a_{0} F(t)+a_{1} F(t-h)+\cdots+a_{n-1} F(t-(n-1) h)\right) & \\
& -\int_{t}^{t+h} F(\tau) d \tau
\end{aligned}
$$

This error function is linear in $F(t)$ and hence if it is zero for $F(t)=e^{r i t}$; $i=1, \cdots, n$, it will be zero for any $F$ that is a linear combination of these exponentials. The condition that $e(t)$ be zero for $F=e^{y_{i t}}$ can be written in the form

$$
a_{0}+a_{1} e^{-\gamma_{i} h}+\cdots+a_{n-1} e^{-r_{i}(n-1) h}=\left(e^{\gamma_{i} h}-1\right)\left(\nu_{i} h\right)^{-1}
$$

when one cancels out $e^{\gamma_{i t}}$ and divides by $h$. Letting $x_{i}=e^{-v_{i h}}$, one obtains the equation

$$
a_{0}+a_{1} x_{i}+a_{2} x_{i}^{2}+\cdots+a_{n-1} x_{i}^{n-1}=-\left(1-x_{i}\right) /\left\{x_{i} \ln x_{i}\right\} .
$$

These equations for $n$ different $\nu_{i}$ will yield $n$ linearly independent equations in $a_{0}, a_{1}, \cdots, a_{n-1}$ since the determinant of this system is the Vander- 
mondian

$$
\left|\begin{array}{ccc}
1, x_{1}, \cdots, x_{1}^{n-1} \\
\cdot \cdot \cdot \cdot \cdot & \cdot \cdot \\
1, x_{n}, \cdots, x_{n}{ }^{n-1}
\end{array}\right|=\prod_{i>j}\left(x_{i}-x_{j}\right)
$$

Notice that the $a_{i}$ depend only on the product $\nu_{i} h$.

In the step by step integration procedure an open integration is used first to obtain from the function values at $t, t-h, \cdots, t-(n-1) h$, a set of values of the unknowns $z_{i}$ and $f_{i}$ at $t=t+h$. One uses the new values at $t+h$ in a "closed" integration formula to recompute $\int_{t}^{t+h} F(\tau) d \tau$. While this method may have certain disadvantages in general, the authors have employed alternate open and closed steps to obtain a simple estimate of the truncation error with a minimum of computation. ${ }^{3}$ In a closed integration step $F(t+h)$ is assumed to be known and the approximating function to $\int_{t}^{t+h} F(\tau) d \tau$ is $h\left[b_{0} F(t+h)+b_{1} F(t)+\cdots+b_{n-1} F(t-(n-2) h)\right]$. Thus the error of the closed procedure $e_{c}(t)$ may be written

$$
\begin{aligned}
e_{c}(t)=h\left[b_{0} F(t+h)+b_{1} F(t)+\cdots+b_{n-1} F(t-(n-2) h)\right] & \\
& -\int_{t}^{t+h} F(\tau) d \tau .
\end{aligned}
$$

The $b_{i}$ are chosen under the assumption that the error is zero for $F(t)=e^{v_{i} t} ; i=1,2, \cdots, n$. This leads to the system of equations

$$
b_{0}+b_{1} e^{-r_{i} h}+\cdots+b_{n-1} e^{-r_{i}(n-1) h}=\left(1-e^{-v_{i} h}\right)\left(\nu_{i} h\right)^{-1} .
$$

Unless otherwise stated the subsequent discussion refers to the open integration procedure.

2. One may wish to consider the same value $\nu_{i}$ more than once in the enumeration of the $\nu_{i}$. For instance, one might want a certain frequency $\nu_{0}$ to appear three times among the $\nu_{i}$, i.e., one might set $\nu_{0}=\nu_{1}=\nu_{2}=\nu_{3}$. It will be indicated below that this will yield superior accuracy for frequencies near $\nu_{0}$. Higher order contacts for various frequencies may be obtained as follows. Suppose in equation (3), $F(t)=e^{\lambda t}$. Let the error $e(t)$ be expressed in the form $h \epsilon(\lambda h) e^{\lambda t}$. Then by certain obvious cancellations, equation (3) yields

$$
\epsilon(\lambda h)=a_{0}+a_{1} e^{-\lambda h}+\cdots+a_{n-1} e^{-\lambda(n-1) h}-\left(e^{\lambda h}-1\right)(\lambda h)^{-1} .
$$

The previous discussion established $\epsilon(\lambda h)=0$ for $\lambda=\nu_{1}, \nu_{2}, \cdots, \nu_{n}$. Now for a double $\nu$ it is clear that $\epsilon(\nu h)=0$ and $\left.\frac{\partial}{\partial \lambda} \epsilon(\lambda h)\right]_{\lambda=\nu}=0$. $\epsilon(\lambda h)$ may be considered a function of $u$ where $u=\lambda h$ and $\frac{\partial \epsilon}{\partial \lambda}=h \frac{d}{d u} \epsilon(u)=h \epsilon^{\prime}(u)$. Thus the partial derivative condition is equivalent to $d \epsilon(u) / d u=0$.

Other functions of $u$ may be used as independent variables and here it is quite convenient to introduce $x=e^{-u}$. Then

$$
\epsilon(x)=a_{0}+a_{1} x+\cdots+a_{n-1} x^{n-1}+f(x)
$$


where

$$
f(x)=(1-x)(x \ln x)^{-1}
$$

The condition $d \epsilon / d u=0$ is equivalent to $d \epsilon / d x=0$, and the system of equations $d \epsilon / d u=0, d^{2} \epsilon / d u^{2}=0$ is equivalent to $d \epsilon / d x=0$ and $d^{2} \epsilon / d x^{2}=0$. Similarly if $\nu_{0}=\nu_{1}=\nu_{2}=\nu_{3}$, let $x_{i}=e^{-\nu_{i} h}$. The first three equations $\epsilon\left(x_{1}\right)=0, \epsilon\left(x_{2}\right)=0, \epsilon\left(x_{3}\right)=0$ of type $\left(6^{\prime}\right)$ may be replaced by the three equations $\epsilon(x)=0, d \epsilon / d x=0, d^{2} \epsilon / d x^{2}=0$, at $x=x_{1}$. Hence, we may use the three equations

$$
\begin{aligned}
& a_{0}+a_{1} x_{1}+\cdots+a_{n-1} x_{1}{ }^{n-1}=-f\left(x_{1}\right), \\
& a_{1}+2 a_{2} x_{1}+\cdots+a_{n-1}(n-1) x_{1}{ }^{n-2}=-f^{\prime}\left(x_{1}\right), \\
& 2 a_{2}+6 a_{3} x_{1}+\cdots+a_{n-1}(n-1)(n-2) x_{1}{ }^{n-3}=-f^{\prime \prime}\left(x_{1}\right),
\end{aligned}
$$

to determine the $a_{i}$ instead of the first three equations of $\left(4^{\prime}\right)$. It is clear how multiplicities in frequencies other than $x_{1}$ can be taken care of by similar methods. One can readily show that the determinant of the new system is not zero. ${ }^{4}$

The use of the equations (8) is generally the most convenient way to determine the $a_{i}$ when they are subject to a multiplicity condition. The conditions

$$
\epsilon(u)=0, \frac{d \epsilon}{d u}=0, \cdots, \frac{d^{n-1} \epsilon}{d u^{n-1}}=0 \text { for } u=0,
$$

are readily seen to be precisely the condition that $e(t)=0$ for $F=1, t, t^{2}$, $\cdots, t^{n-1}$. Thus the polynomial case is included in the above discussion provided multiplicities are considered. The final results of this paper are not affected by letting a number of the frequencies coincide and from the practical point of view, multiple frequencies may be used whenever it is convenient.

3. The foregoing method of integration can be effectively applied to functions of the form

$$
F(t)=\sum_{j=1}^{r} C_{j} e^{\lambda_{j} t}+\sigma(t)
$$

where $\sigma(t)$ is small but otherwise arbitrary. From equation (3) it is seen that the error made in integrating the function $F$ by the above method is

$$
e(t, F)=h \sum_{j=1}^{r} C_{j} \epsilon\left(\lambda_{j} h\right) e^{\lambda_{j} t}+e(t, \sigma),
$$

the significance of the double argument being clear. $\epsilon\left(\lambda_{j} h\right)$ is given by equation (6).

The error may be estimated by equation (10) provided $\epsilon(\lambda h)$ for $\lambda=\lambda_{1}, \cdots, \lambda_{r}$ and $e(t, \sigma)$ are studied. The terms of the summation are then readily estimated since $\left|e^{\lambda t}\right|$ is given by $\exp (t \operatorname{Re}(\lambda)) . \epsilon(\lambda h)$ is evaluated as follows. Suppose that the integration method is precise for step $h$ and complex frequencies $\nu_{1}, \cdots, \nu_{n}$. Now let

$$
y=e^{-\lambda h}-1, \quad y_{j}=e^{-v_{j} h}-1 \text {. }
$$


Then

$$
\epsilon(\lambda h)=(-1)^{n+1}\left[\prod_{j=1}^{n}\left(y-y_{j}\right)\right]\left(A_{n}+A_{n+1} S_{1, n+1}+A_{n+2} S_{2, n+1}+\cdots\right)
$$

where

$$
S_{k, n+1}=\sum_{\alpha_{0}+\alpha_{1}+\cdots+\alpha_{n}=k} y^{\alpha_{0}} y_{1}^{\alpha_{1}} \cdots y_{n}^{\alpha_{n}}
$$

and the $A_{i}$ are given by the series

$$
y((1+y) \ln (1+y))^{-1}=A_{0}+A_{1} y+A_{2} y^{2}+\cdots .
$$

(Cf. Theorem I of $\$ 7$ below.) The $A_{i}$ may readily be calculated since

$$
y[(1+y) \ln (1+y)]^{-1}=\int_{0}^{1}(1+y)^{-r} d r .
$$

If the integral is expanded by the binomial theorem and integrated term by term, one obtains ${ }^{5}$

$$
A_{n}=\frac{(-1)^{n}}{n !} \int_{0}^{1} r(r+1)(r+2) \cdots(r+(n-1)) d r .
$$

The first eleven values of $A_{i}$ are listed below.

$$
\begin{aligned}
& A_{0}=1 \\
& A_{1}=-.5 \\
& A_{2}=.41667 \\
& A_{3}=-.37500 \\
& A_{4}=.34861 \\
& A_{5}=-.32986
\end{aligned}
$$

$$
\begin{aligned}
& A_{6}=.31559 \\
& A_{7}=-.30422 \\
& A_{8}=.29487 \\
& A_{0}=-.28698 \\
& A_{10}=.28019
\end{aligned}
$$

The $A_{i}$ decrease monotonically in absolute value, and alternate in sign. An expression for an overestimate of

$$
A_{n}+A_{n+1} S_{1, n+1}+A_{n+2} S_{2, n+1}+\cdots
$$

can readily be given. Suppose there is a $Y, 0<Y<1$, such that $|y| \leq Y$ and $\left|y_{j}\right| \leq Y$ for $j=1,2, \cdots, n$. Then Lemma 2 below shows that $\left|S_{k, n+1}\right| \leq\left(\begin{array}{c}n+k \\ n\end{array}\right) Y^{k}$. Then

$$
\begin{aligned}
\mid A_{n}+A_{n+1} S_{1, n+1} & +A_{n+2} S_{2, n+1}+\cdots|\leq| A_{n} \mid(1+(n+1) Y \\
& \left.+(n+1)(n+2)(2 !)^{-1} Y^{2}+\cdots\right)=\left|A_{n}\right|(1-Y)^{-(n+1)} .
\end{aligned}
$$

Thus the error is

$$
|\epsilon(\lambda h)| \leq\left[\prod_{j=1}^{n}\left|y-y_{j}\right|\right]\left|A_{n}\right|(1-Y)^{-n-1} .
$$

However this overestimate may be inconvenient when $Y$ is rather close to unity, in which case the original formula (11) will probably give a much smaller estimate.

We note the effect of the factor $\prod_{j=1}^{n}\left|y-y_{j}\right|$. Since $y-y_{j}=e^{-\lambda h}-e^{-r_{i} h}$, 
if $\lambda h$ and $\nu_{i} h$ are small, this difference is similar to $h\left|\lambda-\nu_{i}\right|$; consequently this factor is essentially $h^{n} \prod_{j=1}^{n}\left|\lambda-\nu_{j}\right|$. The original objective of this investigation was to establish that the error goes to zero like $h^{n+1}$. Otherwise a favorable comparison with the known results on polynomial integration could not be established.

It should be emphasized that the above results hold even when a number of the $\nu_{j}$ and consequently $y_{j}$ are equal. For a $\nu_{0}$ which occurs $r$ times, we have a factor $\left|\lambda-\nu_{0}\right| r$ in $\Pi$, which indicates a small $\epsilon$ for $\lambda$ near $\nu_{0}$.

On the other hand one can also take care of the case in which a particular $\lambda_{j}$ has multiple weight in the expression (9) for $F$. For instance, if $\lambda$ has double weight, one finds a term $C t e^{\lambda t}$ in (9) replacing some term $C_{j} e^{\lambda_{j} t}$. This new term $C t e^{\lambda t}$ can be written $\partial\left(C e^{\lambda t}\right) / \partial \lambda$ and consequently in equation (10) instead of $C_{j} \epsilon\left(\lambda_{j} h\right) e^{\lambda_{j} t}$, there appears

$$
\partial\left(C \epsilon(\lambda h) e^{\lambda t}\right) / \partial \lambda \text {. }
$$

Higher weights for $\lambda$ will correspond to higher partials with respect to $\lambda$ in the obvious manner.

A similar investigation of the closed integration procedure yields the result

$\epsilon_{c}(\lambda h)=(-1)^{n+1}\left[\prod_{j=1}^{n}\left(y-y_{j}\right)\right]\left(B_{n}+B_{n+1} S_{1, n+1}+B_{n+2} S_{2, n+1}+\cdots\right) /(y+1)$

where the $S_{i, n+1}, y, y_{i}$ are defined above. $B_{i}=A_{i}+A_{i-1}, B_{0}=A_{0}$. (Cf. Corollary of Theorem I below.)

The important properties of closed integration are present in this result. It can be shown by a gross approximation that for $n>1,\left|B_{n}\right|<\left|A_{n}\right| /(n-1)$ and $B_{n} A_{n}<0$. For $n-1 \geqslant 1$, the error of the closed integration procedure is materially smaller than the error of the open integration and of opposite sign. Two purposes are thus achieved by following an open integration by a closed integration. The error is reduced and a good step truncation overestimate may be found.

The error effect due to the function $\sigma$ is calculated in Theorem II. An overestimate similar to those above is given in the Corollary.

4. The use contemplated for this type of integration is the solution of a system (1) in which the functions $f_{i}\left(z_{1}, \cdots, z_{n}, t\right)$, when obtained as functions of time, $F_{i}(t)$ are of the form (9). In such a use, the complex frequencies $\lambda_{j}$ are not known until the numerical work has been done. On the other hand, one may have initial estimates of reasonable accuracy and the above results indicate that one might well use these initial estimates either as the $\nu_{i}$ directly or to indicate a region in which the $\nu_{i}$ can be chosen to determine the constants $a_{i}$ used in the integration. This means of course that the set of $\nu_{i}$ must be chosen in advance of the integration. One hopes that the choice of the set of $\nu_{i}$ is such that for each frequency $\lambda$ in the $F_{i}$ there is a $\nu_{j}$ such that the factor $\left|\lambda-\nu_{j}\right|$ will be small. If the set of $\lambda$ has many more elements than the number of $\nu_{j}$ permitted by practical considerations, a multiple $\nu_{j}$ may have to compensate for a number of $\lambda$.

In iterative procedures, an open integration is used to yield the first step values of the unknown $z$ at $t+h$. These $z$ are used to compute the $f$ at 
$t+h$ and these values of $f$ are then used to obtain a new value of the increment in $z$ for the interval $(t, t+h)$. The resulting new values of $z$ may be used to recompute the $f$. This process may be repeated until no significant change occurs. But the result even then is not perfect. ${ }^{3}$ If the $f_{i}$ are complicated, the amount of work involved may be proportional to the number of iteration steps and better accuracy may be available from a procedure of simple open integration doing the same amount of work for the same interval of the independent variable but using a smaller step $h$. Thus, for example, an open step followed by a closed step for $h=\Delta t$ may yield a result less accurate than two open steps of length $h=\Delta t / 2$.

On the other hand, in order to use the closed integration procedure to estimate the truncation error, i.e., to take advantage of the fact that in general the error for the closed integration has the opposite sign to that of the open and much smaller, one need not recompute the $f$. The $f$ obtained by substituting the results of the open integration from $t$ to $t+h$ can be used to obtain the $\Delta z$ increment for the closed step from $t$ to $t+h$ and also the increment for the open integration from $t+h$ to $t+2 h$. After the comparison between the increments $\Delta z$ from the open step and the closed step for the interval $t$ to $t+h$, the closed increment is ignored and the open process is continued.

It also should be pointed out that the true open truncation error is not given by equation (3) under these circumstances but by

$$
T(t)=e(t, F)+\int_{t}^{t+h}\left(F(\tau)-f\left(z_{1}, \cdots, z_{n}, \tau\right)\right) d \tau
$$

where the $f$ is from equation (1) and $z_{1}, \cdots, z_{n}$, are true solutions of the system of differential equations which agree with the computed solutions at $t$. However, in general, for a fixed frequency, $T(t)$ may be obtained from $e\left(t, e^{\lambda t}\right)$ by means of a factor which is close to one when $u=\lambda h$ is small. A similar situation exists for the closed integration truncation error. (Cf. references in footnote 3.) The stability of these integration procedures should also be considered.

5. In the appendices to this paper appears certain supplementary material of an illustrative nature or designed to assist the application of the results. In view of formula (11), above, one would normally require that $|y| \ll 1$ for every frequency $\lambda$ present so that $\epsilon(\lambda h)$ will be small. This yields an upper bound for the $h$ 's which one can use. A quick way of finding this upper bound is indicated in Appendix 1. Since the determinant of the system of equations (4) is small, a straightforward numerical elimination will involve a great loss of accuracy in the computation of the $a_{t}$. Practical formulas for the accurate calculation of the $a_{t}$ are given in Appendix 2 with a method of successive improvement. Appendices 3 and 4 contain numerical results which illustrate the theory.

6. In order to evaluate effectively the error it will be necessary to consider briefly the higher Vandermondians, i.e., determinants of the form

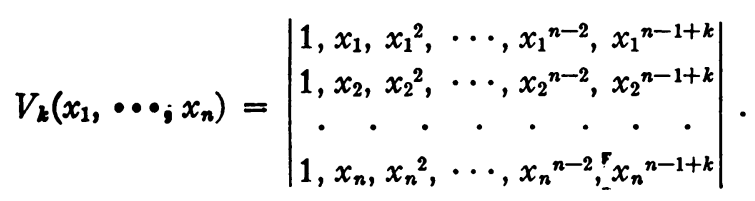


To study this we must consider the result of taking successive divided differences of powers.

We define

$$
\begin{gathered}
{\left[x_{1}, x_{2}\right]_{n}=\left(x_{2}{ }^{n}-x_{1}{ }^{n}\right) /\left(x_{2}-x_{1}\right)} \\
{\left[x_{1}, \cdots, x_{r}\right]_{n}=\left(\left[x_{1}, \cdots, x_{r-1}\right]_{n}-\left[x_{r}, x_{2}, \cdots, x_{r-1}\right]_{n}\right)\left(x_{1}-x_{r}\right)^{-1} .}
\end{gathered}
$$

LEMMA 1. For $r \leq n$,

$$
\left[x_{1}, \cdots, x_{r}\right]_{n}=\sum_{\alpha_{1}+\alpha_{2}+\cdots+\alpha_{r}=n-r+1} x_{1}^{\alpha_{1}} x_{2}^{\alpha_{2}} \cdots x_{r}^{\alpha_{r}}
$$

(in this summation $\left.\alpha_{j}=0,1,2, \cdots\right)$. For $r=n+1$,

$$
\left[x_{1}, \cdots, x_{n+1}\right]_{n}=1
$$

and for $r>n+1$,

$$
\left[x_{1}, \cdots, x_{r}\right]_{n}=0 .
$$

For $r=2$, we see immediately that

$$
\left[x_{1}, x_{2}\right]_{n}=\sum_{\alpha_{1}+\alpha_{2}=n-1} x_{1}^{\alpha_{1}} x_{2}^{\alpha_{2}} \text {. }
$$

In general, if the formula for $r \leq n$ is true for $r-1$, we have

$$
\begin{aligned}
{\left[x_{1}, \cdots, x_{r-1}\right]_{n} } & =\sum_{\alpha_{1}+\cdots+\alpha_{r-1}=n-r+2} x_{1}^{\alpha_{1}} x_{2}^{\alpha_{2}} \cdots x_{r-1}^{\alpha_{r}-1} \\
& =\sum_{\alpha_{1}=0}^{n-r+2} x_{1}^{\alpha_{1}}\left(\sum_{\alpha_{2}+\cdots+\alpha_{r-1=n-r+2-\alpha_{1}}} x_{2}^{\alpha_{2}} \cdots x_{r-1}^{\alpha_{r-1}}\right) \\
& =\sum_{\alpha_{1}=1}^{n-r+2} x_{1}^{\alpha_{1}}\left(\sum_{\alpha_{2}+\cdots+\alpha_{r-1}=n-r+2-\alpha_{1}} x_{2}^{\alpha_{2}} \cdots x_{r-1}^{\alpha_{r-1}}\right) \\
& +\sum_{\alpha_{2}+\cdots+\alpha_{r-1=n-r+2}} x_{2}^{\alpha_{2}} \cdots x_{r-1}^{\alpha_{r-1}}
\end{aligned}
$$

Then we form $\left(\left[x_{r}, x_{2}, \cdots, x_{r-1}\right]_{n}-\left[x_{1}, x_{2}, \cdots, x_{r-1}\right]_{n}\right)\left(x_{r}-x_{1}\right)^{-1}$, $\left[x_{1}, \cdots, x_{r-1}, x_{r}\right]_{n}$

$$
\begin{aligned}
& =\sum_{\alpha_{1}=1}^{n-r+2}\left(\left(x_{r}^{\alpha_{1}}-x_{1}^{\alpha_{1}}\right)\left(x_{r}-x_{1}\right)^{-1}\right) \sum_{\alpha_{2}+\cdots+\alpha_{r-1-n-r+2-\alpha_{1}}} x_{2}^{\alpha_{2}} \cdots x_{r-1}^{\alpha_{r-1}} \\
& =\sum_{\alpha_{1}=1}^{n-r+2}\left(\sum_{\beta_{1}+\beta_{r=\alpha_{1}-1}} x_{1}^{\beta_{1}} x_{r}^{\beta_{r}}\right)\left(\sum_{\alpha_{2}+\cdots+\alpha_{r-1}-n-r+2-\alpha_{1}} x_{2}^{\alpha_{2}} \cdots x_{r-1}^{\alpha_{r-1}}\right) .
\end{aligned}
$$

Every monomial obtained by expanding this expression has

$$
\beta_{1}+\beta_{r}+\alpha_{2}+\cdots+\alpha_{r-1}=n-r+1,
$$

and conversely, every possible term of this type occurs. Consequently for $r \leq n$

$$
\left[x_{1}, \cdots, x_{r-1}, x_{r}\right]_{n}=\sum_{\alpha_{1}+\cdots+\alpha_{r}-n-r+1} x_{1}^{\alpha_{1}} x_{2}^{\alpha_{2}} \cdots x_{r}^{\alpha_{r}} .
$$


To prove the remaining statements, let $r=n$. Then

and

$$
\left[x_{1}, x_{2}, \cdots, x_{n}\right]_{n}=x_{1}+x_{2}+\cdots+x_{n}
$$

$$
\left(\left[x_{n+1}, x_{2}, \cdots, x_{n}\right]_{n}-\left[x_{1}, x_{2}, \cdots, x_{n}\right]_{n}\right)\left(x_{n+1}-x_{1}\right)^{-1}=1 \text {. }
$$

The result for $r>n+1$ is obvious.

The definition of $\left[x_{1}, \cdots, x_{r}\right]_{n}$ can be extended to the cases of multiple $x_{i}$ by means of equations (16), $\left(16^{\prime}\right)$, and $\left(16^{\prime \prime}\right)$.

Lemma 2. For $r \leq n+1$, the number of terms in the expansion of $\left[x_{1}, \cdots, x_{r}\right]_{n}$ is the binomial coefficient $\left(\begin{array}{c}n \\ r-1\end{array}\right)$.

Subject to the condition, $\alpha_{1}+\cdots+\alpha_{r}=n-r+1$, one has as many ways of choosing $\alpha_{1}, \cdots, \alpha_{r}$ as one can arrange $n-r+1$ different things in $r$ groups, blank groups being admissible. This last is the specified binomial coefficient. ${ }^{6}$

Now the higher Vandermondians may be evaluated.

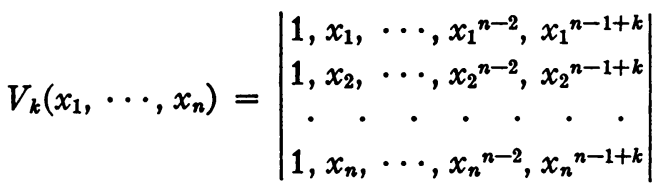

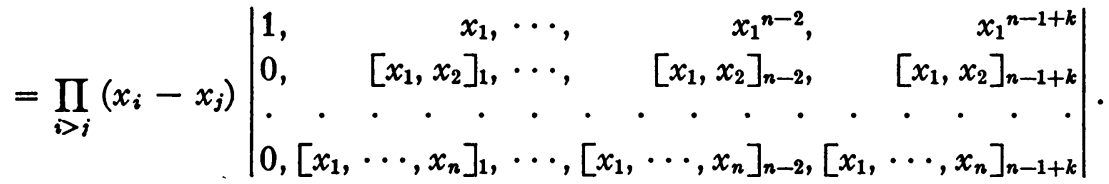

This can readily be seen by subtracting the first row of the original determinant from each of the others and then factoring out $x_{i}-x_{1}$ from the $i$ th row for $i=2, \cdots, n$. One next subtracts the second row from the third, fourth, $\cdots, n$th row and again factors out $x_{i}-x_{2}$ for $i>2$. Continuing this process, one will obtain the displayed result. In the second determinant the terms below the diagonal are zero and all but the corner term of the main diagonal are unity. Thus

\section{LEMMA 3.}

$$
V_{k}\left(x_{1}, \cdots, x_{n}\right)=V_{0}\left(x_{1}, \cdots, x_{n}\right)\left[x_{1}, x_{2}, \cdots, x_{n}\right]_{n-1+k} .
$$

The determinant obtained from a Vandermondian by differentiating a row and setting the variable of this row equal to the variable of another row will be considered. More generally, a determinant may have the same variable in a number of rows each of which, except the uppermost, is obtained by differentiating the one above it. For these a notation is introduced which may be illustrated.

$$
\begin{aligned}
& V_{k}\left(\left\{x_{1}\right\}^{(3)},\left\{x_{2}\right\}^{(2)}, \cdots\right)
\end{aligned}
$$

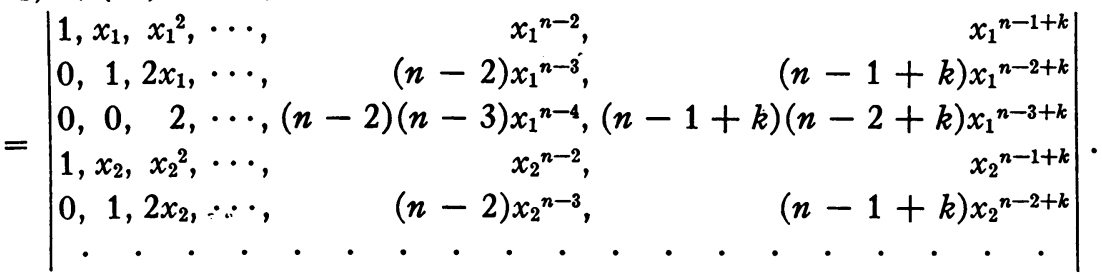


It is clear, for instance, that

and that

$$
\left.V_{k}\left(\left\{x_{1}\right\}^{(3)}, x_{4}, \cdots, x_{n}\right)=\frac{\partial^{3}}{\partial x_{3}^{2} \partial x_{2}} V_{k}\left(x_{1}, \cdots, x_{n}\right)\right]_{x_{2}=x_{1}, x_{3}=x_{1}}
$$

and

$$
\left.V_{0}\left(x_{1}, \cdots, x_{n}\right)\right]_{x_{2}=x_{1}, x_{3}=x_{1}}=0
$$

$$
\begin{aligned}
&\left.\frac{\partial}{\partial x_{2}} V_{0}\right]_{x_{2}=x_{1}, x_{3}-x_{1}}\left.=0, \quad \frac{\partial}{\partial x_{3}} V_{0}\right]_{x_{2}-x_{1}, x_{3}-x_{1}}=0 \\
&\left.\left.\frac{\partial^{2}}{\partial x_{2} \partial x_{3}} V_{0}\right]_{x_{2}-x_{1}, x_{2}-x_{1}}=0, \quad \frac{\partial^{2}}{\partial x_{3}^{2}} V_{0}\right]_{x_{2}-x_{1}, x_{3}-x_{1}}=0 .
\end{aligned}
$$

Consequently when $\frac{\partial^{3}}{\partial x_{3}{ }^{2} \partial x_{2}}$ is applied to both sides of the equation (18) and then one lets $x_{2}=x_{1}$ and $x_{3}=x_{1}$, all but one of the terms on the right hand side drop out. This leads to the equation

$$
V_{k}\left(\left\{x_{1}\right\}^{(3)}, x_{4}, \cdots, x_{n}\right)=V_{0}\left(\left\{x_{1}\right\}^{(3)}, x_{4}, \cdots, x_{n}\right)\left[x_{1}, x_{1}, x_{1}, x_{4}, \cdots, x_{n}\right]_{n-1+k} .
$$

This argument generalizes; hence

\section{LEMMA 4.}

$$
\begin{aligned}
& V_{k}\left(\left\{x_{1}\right\}^{\left(p_{1}\right)},\left\{x_{2}\right\}^{\left(p_{2}\right)}, \cdots\right) \\
& \quad=V_{0}\left(\left\{x_{1}\right\}^{\left(p_{1}\right)},\left\{x_{2}\right\}^{\left(p_{2}\right)}, \cdots\right)\left[x_{1}, x_{1}, \cdots, x_{2}, x_{2}, \cdots\right]_{n-1+k}
\end{aligned}
$$

where $x_{j}$ occurs $p_{j}$ times in the brackets.

7. Consider now determinants similar to the Vandermondians except that a column is omitted. The following notation will be used.

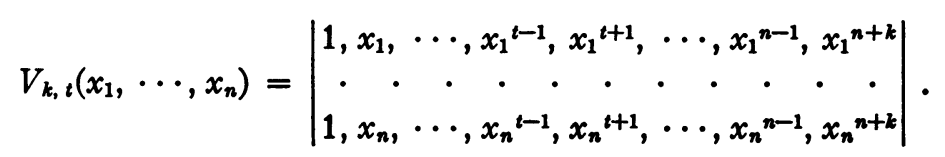

Form

$$
\begin{aligned}
\sum_{t=0}^{n-1}(-1)^{t} y^{t} V_{k, t}\left(x_{1},\right. & \left.\cdots, x_{n}\right)+(-1)^{n} y^{n+k} V_{0, t}\left(x_{1}, \cdots, x_{n}\right) \\
& =V_{k}\left(y, x_{1}, \cdots, x_{n}\right)=V_{0}\left(y, x_{1}, \cdots, x_{n}\right)\left[y, x_{1}, \cdots, x_{n}\right]_{n+k} .
\end{aligned}
$$

Now if $p_{t}\left(x_{1}, \cdots, x_{n}\right)$ is the elementary symmetric function of degree $t$ in $x_{1}, x_{2}, \cdots, x_{n}{ }^{7}$

$$
V_{0}\left(y, x_{1}, \cdots, x_{n}\right)=V_{0}\left(x_{1}, \cdots, x_{n}\right) \sum_{t=0}^{n}(-1)^{t} p_{n-t} y^{t} .
$$

On the other hand

$$
\begin{aligned}
{\left[y, x_{1}, \cdots, x_{n}\right]_{n+k} } & =\sum_{\alpha_{0}+\alpha_{1}+\cdots+\alpha_{n}=k} y^{\alpha_{0}} x_{1}{ }^{\alpha_{1}} \cdots x_{n}^{\alpha_{n}} \\
& =\sum_{\alpha=0}^{k} y^{\alpha} S_{k-\alpha, n}
\end{aligned}
$$


where

$$
S_{m, n}=\sum_{\alpha_{1}+\cdots+\alpha_{n}=m} x_{1}^{\alpha_{1}} \cdots x_{n}^{\alpha_{n}}
$$

Hence

$$
\begin{aligned}
V_{k}\left(y, x_{1}, \cdots, x_{n}\right)= & V_{0}\left(y, x_{1}, \cdots, x_{n}\right)\left[y, x_{1}, \cdots, x_{n}\right]_{n+k} \\
& =V_{0}\left(x_{1}, \cdots, x_{n}\right)\left(\sum_{\beta=0}^{n}(-1)^{\beta} p_{n-\beta} y^{\beta} \sum_{\alpha=0}^{k} y^{\alpha} S_{k-\alpha, n}\right) \\
& =V_{0}\left(x_{1}, \cdots, x_{n}\right)\left(\sum_{\gamma=0}^{n+k} \sum_{\beta=\max (0, \gamma-k)}^{\min (\gamma, n)}(-1)^{\beta} p_{n-\beta} S_{k-\gamma+\beta, n}\right) y^{\gamma} .
\end{aligned}
$$

Thus for $0 \leq t \leq n-1$,

$$
V_{k, t}\left(x_{1}, \cdots, x_{n}\right)=V_{0}\left(x_{1}, \cdots, x_{n}\right)(-1)^{t} \sum_{\beta=\max (0, t-k)}^{t}(-1)^{\beta} p_{n-\beta} S_{k+\beta-t, n} .
$$

Since the previous discussion of multiple frequencies also applies here we have

\section{LEMMA 5.}

$$
\begin{aligned}
V_{k, t}\left(\left\{x_{1}\right\}^{\left(p_{1}\right)}, \cdots,\left\{x_{r}\right\}^{\left(p_{r}\right)}\right) \\
\quad=V_{0}\left(\left\{x_{1}\right\}^{\left(p_{1}\right)}, \cdots,\left\{x_{r}\right\}^{\left(p_{r}\right)}\right) \sum_{\beta=\max (0, t-k)}^{t}(-1)^{\beta+t} p_{n-\beta} S_{k-t+\beta, n} .
\end{aligned}
$$

8. The error in using the formula $h\left[a_{0} F(t)+\cdots+a_{n-1} F(t-(n-1) h)\right]$ for $\int_{t}^{t+h} F(t) d t$ can be explicitly computed for the case $F(t)$ of the form $e^{\lambda t}$.

Suppose that this error $e\left(t, e^{\lambda t}\right)$ is in the form $h \epsilon(\lambda h) e^{\lambda t}$. Then,

$$
\begin{aligned}
h\left[a_{0} e^{\lambda t}+a_{1} e^{\lambda(t-h)}+\cdots+a_{n-1} e^{\lambda(t-(n-1) h)}\right]-h \epsilon(\lambda h) e^{\lambda t} & \\
& =\lambda^{-1}\left(e^{\lambda(t+h)}-e^{\lambda t}\right) .
\end{aligned}
$$

A corresponding equation holds for $\lambda=\nu_{i}, i=1, \cdots, n$, with $\epsilon\left(\nu_{i} h\right)=0$. Cancel the $e^{\lambda t}$ from equation (23) and $e^{\nu_{i t}}$ from the corresponding equations in the $\nu_{i}$. Then

$$
\begin{aligned}
& a_{0}+a_{1} e^{-\lambda h}+\cdots+a_{n-1} e^{-(n-1) \lambda h}-\epsilon(\lambda h)=(h \lambda)^{-1}\left(e^{\lambda h}-1\right) \\
& a_{0}+a_{1} e^{-\eta_{1} h}+\cdots+a_{n-1} e^{-(n-1) \nu_{1} h}=\left(h \nu_{1}\right)^{-1}\left(e^{\nu_{1} h}-1\right) \\
& a_{0}+a_{1} e^{-\nu_{n} h}+\cdots+a_{n-1} e^{-(n-1) v_{n} h} \quad=\left(h \nu_{n}\right)^{-1}\left(e^{\nu_{n} h}-1\right)
\end{aligned}
$$

It is convenient to introduce $x=e^{-\lambda h}, x_{i}=e^{-v_{i} h}$ and $f(x)=(1-x)(x \ln x)^{-1}$. The above system of equations is then solved for $\epsilon(\lambda h)$.

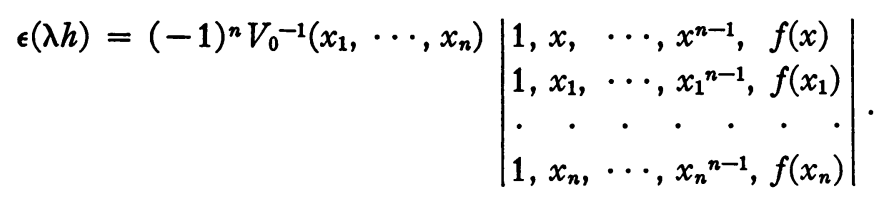

Let $x-1=y, x_{j}-1=y_{j}$. Since $V_{0}\left(x_{1}, \cdots, x_{n}\right)$ depends only on $x_{i}-x_{j}, V_{0}\left(x_{1}, \cdots, x_{n}\right)=V_{0}\left(y_{1}, \cdots, y_{n}\right)$. The $x_{i}$ in the first $n$ columns of 
the second determinant may be replaced by $y_{i}$, by simple manipulations on determinants.

Let $g(y)=y((1+y) \ln (1+y))^{-1}$. Thus $f(x)=-g(y)$ and

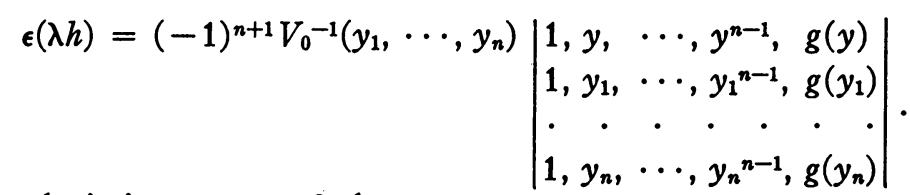

$g(y)$ is analytic in $y$ at $y=0$; hence

$$
g(y)=A_{0}+A_{1} y+A_{2} y^{2}+\cdots .
$$

When this expression for $g(y)$ and the analogous expression for $g\left(y_{j}\right)$ are inserted above, the elementary properties of determinants permit us to cancel out the first $n$ terms in the $g$ series. If the distributive property of the last column is used, one obtains for $|y|<1$ and $\left|y_{j}\right|<1$,

$$
\begin{aligned}
\epsilon(\lambda h)= & (-1)^{n+1} V_{0}^{-1}\left(y_{1}, \cdots, y_{n}\right)\left(A_{n} V_{0}\left(y, y_{1}, \cdots, y_{n}\right)\right. \\
& \left.\quad+A_{n+1} V_{1}\left(y, y_{1}, \cdots, y_{n}\right)+\cdots+A_{n+k} V_{k}\left(y, y_{1}, \cdots, y_{n}\right)+\cdots\right) \\
= & (-1)^{n+1}\left(\frac{V_{0}\left(y, y_{1}, \cdots, y_{n}\right)}{V_{0}\left(y_{1}, \cdots, y_{n}\right)}\right)\left(A_{n}+A_{n+1} S_{1, n+1}+A_{n+2} S_{2, n+1}+\cdots\right) \\
= & (-1)^{n+1}\left[\Pi\left(y-y_{j}\right)\right]\left(A_{n}+A_{n+1} S_{1, n+1}+A_{n+2} S_{2, n+1}+\cdots\right),
\end{aligned}
$$

when we use LEMma 3 and let

$$
S_{k, n+1}=\sum_{\alpha_{0}+\alpha_{1}+\cdots+\alpha_{n}=k} y^{\alpha_{0}} y_{1}^{\alpha_{1}} \cdots y_{n}^{\alpha_{n}} .
$$

The $A_{n}$ are readily calculated from the expression for $g$,

$$
g(y)=\frac{y}{(1+y) \ln (1+y)}=\int_{0}^{1}(1+y)^{-r} d r .
$$

If the integral is expanded by the binomial theorem and integrated term by term,

$$
A_{n}=\frac{(-1)^{n}}{n !} \int_{0}^{1} r(r+1) \cdots(r+[n-1]) d r .
$$

The values up to $A_{10}$ have been given in section 3 .

THEOREM I. Let $a_{0}, a_{1}, \cdots, a_{n-1}$ be chosen so that

$$
h\left[a_{0} F(t)+a_{1} F(t-h)+\cdots+a_{n-1} F(t-(n-1) h)\right]=\int_{t}^{t+h} F(\tau) d \tau
$$

when $F(t)=e^{\nu_{i} t}$ for $i=1, \cdots, n$. Let $e(t, F)$ denote

$$
e(t, F)=h\left[a_{0} F(t)+a_{1} F(t-h)+\cdots+a_{n-1} F(t-(n-1) h)\right]-\int_{t}^{t+h} F(\tau) d \tau .
$$

Then for $F=e^{\lambda t}$ and $e(t, F)=h \epsilon(\lambda h) e^{\lambda t}$,

$\epsilon(\lambda h)=(-1)^{n+1}\left[\Pi\left(y-y_{j}\right)\right]\left(A_{n}+A_{n+1} S_{1, n+1}+\cdots+A_{n+k} S_{k, n+1}+\cdots\right)$ where $y=e^{-\lambda h}-1, y_{j}=e^{-r j h}-1$, provided $|y|<1$ and $\left|y_{j}\right|<1$, and

$$
S_{k, n+1}=\sum_{\alpha_{0}+\alpha_{1}+\cdots+\alpha_{n-k}} y^{\alpha_{0}} y_{1}^{\alpha_{1}} \cdots y_{n}^{\alpha_{n}} .
$$


The case of multiple frequencies is obtained by direct substitution. so that

Corollary. (Closed integration theorem.) Let $b_{0}, b_{1}, \cdots, b_{n-1}$ be chosen $h\left[b_{0} F(t+h)+b_{1} F(t)+b_{2} F(t-h)+\cdots+b_{n-1} F(t-(n-2) h)\right]=\int_{t}^{t+h} F(\tau) d \tau$ when $F(t)=e^{r i t}$ for $i=1,2, \cdots, n$. Let $e_{0}(t, F)$ denote $e_{c}(t, F)=h\left[b_{0} F(t+h)+b_{1} F(t)+b_{2} F(t-h)+\cdots\right.$

$$
+b_{n-1}(F(t-(n-2) h)]-\int_{t}^{t+h} F(\tau) d \tau \text {. }
$$

Then for $F=e^{\lambda t}$ and $e_{c}(t, F)=h \epsilon_{c}(\lambda h) e^{\lambda t}$,

$$
\begin{aligned}
\epsilon_{c}(\lambda h)=(-1)^{n+1} e^{\lambda h} \Pi\left(y-y_{j}\right)\left(B_{n}+B_{n+1} S_{1, n+1}\right. & +\cdots \\
& +B_{n+k} S_{k, n+1}
\end{aligned}
$$

where the $y$ and $y_{j}$ are as defined above, and

$$
B_{k}=A_{k}+A_{k-1} \text {. }
$$

The discussion for closed integration is analogous to that given above for open integration, except that one has an additional factor $e^{\lambda h}$ in the coefficients of the unknowns in the equivalent of equations (24). It is desirable to divide by this factor and solve for $e^{-\lambda h_{c}}(\lambda h)$. As a result of this division $f_{c}$ becomes $(1-x) / \ln x$ and $g_{c}(y)$ is $y / \ln (1+y)$. The result $\epsilon_{c}(\lambda h)$ then is obtained by replacing the open $g(y)=g_{0}(y)$ by $g_{c}(y)$ and multi. plying by $e^{\lambda h}$. Thus the $A_{i}$ are to be replaced by the $B_{i}$ which are obtained from

$$
B_{0}+B_{1} y+B_{2} y^{2}+\cdots=y / \ln (1+y) \text {. }
$$

Inasmuch as $(1+y) g_{0}(y)=g_{c}(y)$ it follows that

$$
B_{n}=A_{n}+A_{n-1}=(-1)^{n} \int_{0}^{1} \frac{r(r+1) \cdots(r+(n-1))}{n !}\left[1-\frac{n}{r+(n-1)}\right] d r \text {. }
$$

Thus $B_{n}$ is opposite in sign to $A_{n}$, since $0 \geq \frac{r-1}{n+r-1} \geq-\frac{1}{n-1}$, and $\left|B_{n}\right| \leq \frac{1}{n-1}\left|A_{n}\right|$.

9. An expression is needed to represent the error $e(t, \sigma)=s h$ which occurs when the function $\sigma(t)$ is openly integrated. The following process will not be carried through for a closed integration procedure. Let $\sigma_{0}=\sigma(t)$, $\sigma_{1}=\sigma(t-h)$, etc. Then

$$
h\left(a_{0} \sigma_{0}+a_{1} \sigma_{1}+\cdots+a_{n-1} \sigma_{n-1}-s\right)=\int_{t}^{t+h} \sigma(\tau) d \tau=-s^{\prime \prime} h .
$$

This equation and the set $\left(4^{\prime}\right)$ permit one to solve for $a_{i}$ and $s$.

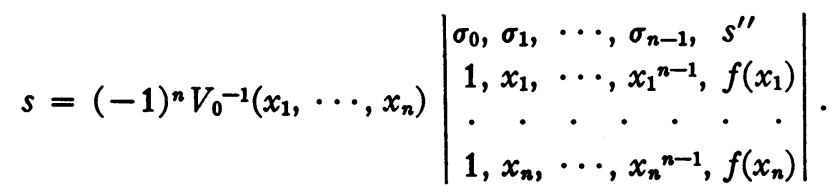


To introduce the $y_{i}$, let $\eta_{0}=\sigma_{0}, \eta_{1}=\sigma_{1}-\sigma_{0}, \eta_{2}=\sigma_{2}-2 \sigma_{1}+\sigma_{0}$ and in general

$$
\eta_{k}=\sigma_{k}-k \sigma_{k-1}+\left(\begin{array}{l}
k \\
2
\end{array}\right) \sigma_{k-2}+\cdots+(-1)^{k} \sigma_{0} .
$$

Note that if the $\sigma$ alternate in sign and are all equal in absolute value then $\eta_{k}=2^{k} \sigma$. With this notation,

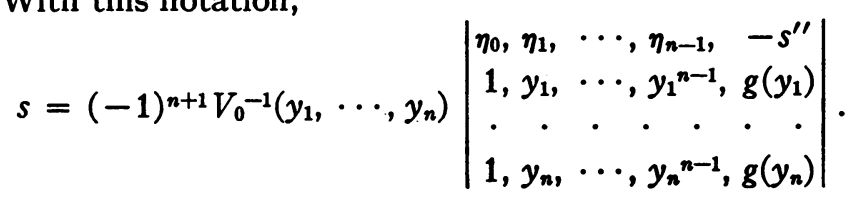

If the determinant is expanded in terms of the first row, $s^{\prime \prime}$ appears multiplied by $V_{0}\left(y_{1}, \cdots, y_{n}\right)$. However, $\eta_{\alpha}$ is multiplied by a determinant without an $\alpha$ power column of the $y_{i}$ but with the $g$ column. Expanding the $g_{i}$ of this column in a $y$ series the $\alpha$ column cannot be cancelled as before, but instead one obtains the Vandermondian itself. Thus

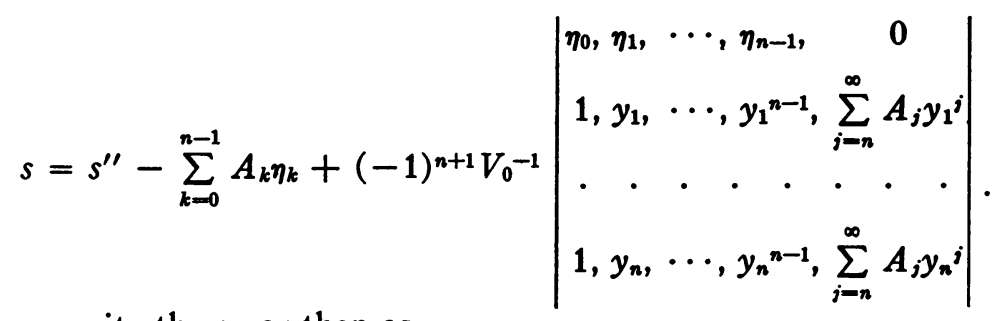

We may write the error then as

$$
s=s^{\prime \prime}-\sum_{k=0}^{n-1} A_{k} \eta_{k}+(-1)^{n+1} \sigma^{\prime} .
$$

Let

$$
D_{\beta}=\sum_{k=\beta}^{n-1}(-1)^{k+\beta} A_{k}\left(\begin{array}{l}
k \\
\beta
\end{array}\right)
$$

Since

$$
\begin{aligned}
& \eta_{k}=\sum_{\beta=0}^{k}(-1)^{k-\beta}\left(\begin{array}{l}
k \\
\beta
\end{array}\right) \sigma_{\beta}, \\
& s=s^{\prime \prime}-\sum_{\beta=0}^{n-1} D_{\beta} \sigma_{\beta}+(-1)^{n+1} \sigma^{\prime} .
\end{aligned}
$$

Now

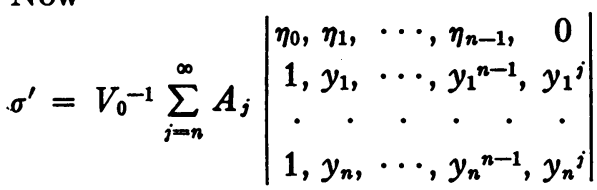

$$
\begin{aligned}
& =\sum_{j=n}^{\infty} A_{j} \sum_{t=0}^{n-1}(-1)^{t} \eta_{t} V_{0}^{-1} V_{j-n, t}
\end{aligned}
$$

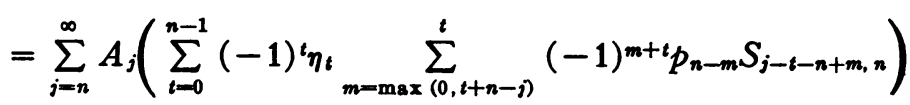


when LEMma 5 is used. Then

$$
\sigma^{\prime}=p_{n} \eta_{0} \sum_{j=n}^{\infty} A_{j} S_{j-n, n}+\sum_{t=1}^{n-1} \eta_{t} \sum_{m=0}^{t}(-1)^{m} p_{n-m} \sum_{j=n+t-m}^{\infty} A_{j} S_{j-t-n+m, n} .
$$

Define

$$
G_{r}=\sum_{k=0}^{\infty} A_{r+k} S_{k, n}
$$

Then

$$
\sigma^{\prime}=p_{n} \eta_{0} G_{n}+\sum_{t=1}^{n-1} \eta_{t} \sum_{m=0}^{t}(-1)^{m} p_{n-m} G_{n-m+t}
$$

Thus if

$$
T_{n, t}=\sum_{m=0}^{t}(-1)^{m} p_{n-m} G_{n+t-m}
$$

we have

$$
\sigma^{\prime}=\sum_{t=0}^{n-1} \eta_{t} T_{n, t}
$$

since $T_{n, 0}=p_{n} G_{n}$. It was shown that

$$
\eta_{t}=\sum_{\beta=0}^{t}(-1)^{t-\beta}\left(\begin{array}{c}
t \\
\beta
\end{array}\right) \sigma_{\beta}
$$

hence

$$
\begin{aligned}
\sigma^{\prime} & =\sum_{t=0}^{n-1} \sum_{\beta=0}^{t}(-1)^{t-\beta}\left(\begin{array}{c}
t \\
\beta
\end{array}\right) \sigma_{\beta} T_{n, t} \\
& =\sum_{\beta=0}^{n-1} \sum_{t=\beta}^{n-1}\left(\begin{array}{c}
t \\
\beta
\end{array}\right) T_{n, t}(-1)^{t-\beta} \sigma_{\beta} \\
& =\sum_{\beta=0}^{n-1} H_{n, \beta}(-1)^{\beta} \sigma_{\beta} \text { where } H_{n, \beta}=\sum_{t=\beta}^{n-1}(-1)^{t}\left(\begin{array}{c}
t \\
\beta
\end{array}\right) T_{n, t} .
\end{aligned}
$$

THEOREM II. If

$$
\begin{gathered}
S_{k, n}=\sum_{\alpha_{1}+\cdots+\alpha_{n}=k} y_{1}^{\alpha_{1} \cdots y_{n} \alpha_{n},} \\
G_{r}=\sum_{k=0}^{\infty} A_{r+k} S_{k, n},
\end{gathered}
$$

$p_{\mu}$ is the elementary symmetric function of degree $\mu$ in $y_{1}, \cdots, y_{n}$,

$$
\begin{aligned}
T_{n, t} & =\sum_{m=0}^{t}(-1)^{m} p_{n-m} G_{n+t-m}, \\
H_{n, \beta} & =\sum_{t=\beta}^{n-1}(-1)^{t}\left(\begin{array}{l}
t \\
\beta
\end{array}\right) T_{n, t}, \\
D_{k} & =\sum_{r=k}^{n-1}(-1)^{k+r} A_{r}\left(\begin{array}{l}
r \\
k
\end{array}\right),
\end{aligned}
$$


then the error in integrating an arbitrary function $\sigma$ is given by

where

$$
e=h s=h\left[\frac{1}{h} \int_{t}^{t+h} \sigma d t+\sum_{k=0}^{n-1}(-1)^{k} D_{k} \sigma_{k}+\sigma^{\prime}\right]
$$

and

$$
\eta_{k}=\sum_{\beta=0}^{k}(-1)^{k-\beta}\left(\begin{array}{l}
k \\
\beta
\end{array}\right) \sigma_{\beta}
$$

$$
\sigma^{\prime}=\sum_{t=0}^{n-1} \eta_{t} T_{n, t}=\sum_{\beta=0}^{n-1} H_{n, \beta}(-1)^{\beta} \sigma_{\beta}
$$

$\sigma^{\prime}$ can now be overestimated as follows :

Let $Y, 0<Y<1$ be a bound on $\left(|y|,\left|y_{i}\right|\right) .\left|A_{k}\right| \leq 1$. Hence, from the definition of $G_{r}$, and the result of Lemma 2

$$
\left|G_{r}\right| \leq \sum_{k=0}^{\infty}\left(\begin{array}{c}
k+n-1 \\
n-1
\end{array}\right) Y^{k}=\frac{1}{(1-Y)^{n}} .
$$

This is a bound independent of $r$. As $r$ increases, the bound may be improved by the factor $\left|A_{r}\right|$.

Now $\left|T_{n, t}\right|$ is also bounded.

$$
\begin{aligned}
\left|T_{n, t}\right| & \leq \sum_{m=0}^{t}\left|p_{n-m}\right| \frac{1}{(1-Y)^{n}} \\
& \leq \sum_{m=0}^{t}\left(\begin{array}{c}
n \\
m
\end{array}\right) \frac{Y^{n-m}}{(1-Y)^{n}}
\end{aligned}
$$

This may be inserted into the equation for $H_{n, \beta}$,

$$
\begin{aligned}
\left|H_{n, \beta}\right| & \leq \sum_{t=\beta}^{n-1}\left(\begin{array}{c}
t \\
\beta
\end{array}\right) \sum_{m=0}^{t} \frac{Y^{n-m}}{(1-Y)^{n}} \\
& =\sum_{m=0}^{n-1}\left(\begin{array}{c}
n \\
m
\end{array}\right) \frac{Y^{n-m}}{(1-Y)^{n}} \sum_{t=\max (\beta, m)}^{n-1}\left(\begin{array}{l}
t \\
\beta
\end{array}\right) .
\end{aligned}
$$

Since $\left(\begin{array}{c}t \\ \beta\end{array}\right)=\left(\begin{array}{c}t+1 \\ \beta+1\end{array}\right)-\left(\begin{array}{c}t \\ \beta+1\end{array}\right)$,

Thus

$$
\left|H_{n, \beta}\right| \leq \sum_{m=0}^{n-1}\left(\begin{array}{c}
n \\
m
\end{array}\right) \frac{Y^{n-m}}{(1-Y)^{n}}\left[\left(\begin{array}{c}
n \\
\beta+1
\end{array}\right)-\left(\begin{array}{c}
n-\max (\beta, t) \\
\beta+1
\end{array}\right)\right] .
$$

Now

$$
\left|H_{n, \beta}\right| \leq \frac{\left(\begin{array}{c}
n \\
\beta+1
\end{array}\right)}{(1-Y)^{n}} \sum_{m=0}^{n-1}\left(\begin{array}{c}
n \\
m
\end{array}\right) Y^{n-m}=\left(\begin{array}{c}
n \\
\beta+1
\end{array}\right) \frac{(Y+1)^{n}-1}{(1-Y)^{n}}
$$

$$
\sigma^{\prime}=\sum_{\beta=0}^{n-1} H_{n, \beta}(-1)^{\beta} \sigma_{\beta}
$$


Let $\sigma=\max \left|\sigma_{i}\right|$, then

$$
\left|\sigma^{\prime}\right|<\frac{\left(2^{n}-1\right) \sigma\left[(Y+1)^{n}-1\right]}{(1-Y)^{n}} .
$$

COROLlary. The error $e(t, \sigma)$ of integrating a function with a maximum value of $\sigma$ is bounded by

$$
|e(t, \sigma)| \leq \sigma h\left[2^{n}+\frac{(Y+1)^{n}-1}{(1-Y)^{n}}\left(2^{n}-1\right)\right] .
$$

Project Cyclone

P. BROCK

Reeves Instrument Corp., N. Y.

F. J. MurRay

Columbia University

[Editorial Note: A second part of this paper, to appear in the next issue of $M T A C$, will contain illustrative numerical examples of the application of the above ideas.]

1 W. E. MILNE, "The remainder in linear methods of approximation," NBS, Jn. Research, v. 43,1949 , p. 501-511. This gives a more general approach to step errors of integration formulas based upon approximation by sets of functions.

2 R. E. GREENwOOD, "Numerical integration of linear sums of exponential functions," Ann. Math. Stat., v. 20, 1949, p. 608-611.

¿ P. Brock \& F. J. MURRAY, "Planning and error analysis for the numerical solution of a test system of differential equations on the IBM sequence calculator," Cyclone Report, Reeves Instrument Corp., New York 28. See also F. J. MURRAY, "Planning and error considerations for the numerical solution of a system of differential equations on a sequence calculator," $M T A C$, v. 4, p. 133-144.

'F. J. MURRay, "Linear equation solvers," Quart. Appl. Math., v. 7, 1948, p. 263-274.

- L. H. Thomas of the Watson Scientific Computing Laboratory indicated this formula for $A_{n}$ to the authors. He also indicated that the $A_{n}$ are equal in absolute value to the coefficients of the Adams-Bashforth method of step by step numerical integration.

- W. Feller, Probability Theory. New York, 1950, v. 1, p. 52.

7 G. Birkhoff \& S. MacLANE, A Survey of Modern Algebra. New York, 1948, p. 424.

\section{A Note on the Inversion of Matrices by Random Walks}

In a recent note, Forsythe $\&$ LEIBLER ${ }^{1}$ described a method (first suggested by J. v. NeumanN and S. M. Ulam) for the inversion of certain types of matrices by a "Monte Carlo" sampling procedure. The authors explain their scheme in terms of drawing balls from an urn, but the procedure might, of course, be just as well described as a random walk.

A boundary value problem involving a difference equation in a bounded domain is equivalent to a system of linear algebraic equations in as many unknowns as there are lattice points in the domain. It is therefore to be expected that the sampling methods for the solution of such difference equations as explained in CURTISS ${ }^{2}$ and WASOW ${ }^{3}$ are closely related to the method of Forsythe \& Le:bler. ${ }^{1}$

In order to study this relation we rephrase the latter method in the language of random walks. We consider a set of $m$ points $P_{1}, \cdots, P_{m}$ and introduce a moving particle which, starting from $P_{i}$, jumps from point to point in such a way that the probability of going from $P_{\nu}$ to $P_{\mu}$ in one jump is $p_{\nu \mu}$. Also at each point $P_{\nu}$ there is a probability $p_{\nu}=1-\sum_{\mu=1}^{m} p_{\nu \mu}$ of the random walk ending there. 